\title{
Neonatal ichthyosis-sclerosing cholangitis syndrome
}

INSERM

\section{Source}

INSERM. (1999). Orphanet: an online rare disease and orphan drug data base. Neonatal ichthyosis-sclerosing cholangitis syndrome. ORPHA:59303

Neonatal ichthyosis-sclerosing cholangitis (NISCH syndrome) is a very rare complex ichthyosis syndrome characterized by scalp hypotrichosis, scarring alopecia, ichthyosis and sclerosing cholangitis. 